\title{
A Study on Camera Work Extraction Technology from Compressed Image Information
}

\author{
(圧縮した動画像情報からのカメラワーク抽出技術の検討)
}

Yang Yang ${ }^{\dagger}$, Shizuo Nakano (member) ${ }^{\dagger}$

\begin{abstract}
It is important to structure video sequences for efficiently editing and indexing the images that may be requested from an enormous volume of video data. One approach to structuring is to divide a series of images into fixed units such as cut points or camera work, and then retrieving against the units. This paper proposes a method of automatically extracting camera work sequences. Specifically, in this paper, MPEG2, which can be mainstreem in future, coded video data is targeted. To reduce the volume of data to be processed, to eliminate the time need for decoding, and to avoid video loss due to decoding, we estimate the camera work using the extracted motion vectors which used in the motion compensation process. The proposed method extracts the motion vectors using motion compensation. To recognize the camera work in each frame, we also present an algorithm for extracting each camera work sequence. The efficiency of the method is confirmed using MPEG2 coded image data of diverse test video sequences.
\end{abstract}

Key words: MPEG2, Video retrieving and editing, Structured video, Camera work, Motion vector

\section{Introduction}

Given the recent advances in broadband computer networks and high performance personal computers, image and video media are becoming more important. Since video media can provide better human interfaces, the information contained in video has become an important field of research. Video data contains so much information that a longer time is needed for retrieving and editing it. In order to solve this problem, the technology which retrieves correctly and fastly the request image information from the storaged encoded data and edits easily them is needed.

To realize this technology, it is necessary to extract the structure of the video data and create structured video $^{1)}$. To this end, an additional piece of information about the video contents, called a header, is defined and placed it at the begining of the video data. The information in the header describes scene changes, camera work, and so on ${ }^{2 / 3)}$. In the proposed method, when the video data is digitized and compressed, it is structured by the computer at the same time. Since this technology is capable of directly editing compressed video data,

Received March 31, 1999; Revised July 26, 1999; Accepted September 20, 1999

† Accepted Faculty of Engineering, Toyama Prefectural University (Kurokawa 5180, Kosugi-machi, Imizu-gun, Toyama 939-0398, Japan) it can realize rapid and highly efficient editing.

Due to the large amount of data involved, video sequences are often compressed for efficient transmission or storage on-line. However, most current camera work detection techniques operate on uncompressed video sequences $^{4) \sim 6)}$. These compressed video sequences have to go through computationally intensive processing to be de-compressed first before any camera work detection algotithms can be applied. The volume of information of video data compressed by standards like MPEG2 for computer storage, for instance, is only $1 / 20 \sim 1 / 25$ that of the original. Therefore, this paper describes a method of detecting camera work directly from MPEG2 compressed video data. The reasons for this are : i) reducing the volume of data to be processed, ii) eliminating the time need for decoding, and iii) avoiding video loss due to decoding.

Some papers detected the camera work from encoded data $^{7 / 8)}$. They detected the camera work only in each frame and did not refer to the detection of the camera works in whole frame sequence. Originally, because the detection of the camera work such as panning or zooming is the one defining in a time section, it will be a method in which establishment is high using the re-judgment with the time sequence. The characteristic of this paper is that it extracts the camera work in 
a time section using the plural detected frames again. In addition, the presumption algorithm of two types of combination camera works is also proposed.

This paper targets the motion compensation function of the MPEG2 compression standard for extracting the information of camera work. That is, motion vector information is used to detect camera work. Video sequences normally contain global motion and local motion. Global motion is the motion that is impressed on all elements in the frame. It is caused by camera work (See Fig.1). Local motion is object motion. Local motion is usually restricted to parts of each frame (See Fig.2). Each camera work induces a characteristic motion vector pattern in the video sequences. For example, if the camera is panned to the left, background and stationary objects move to the right, and the traces of points generated by the objects can be extracted as motion vectors.

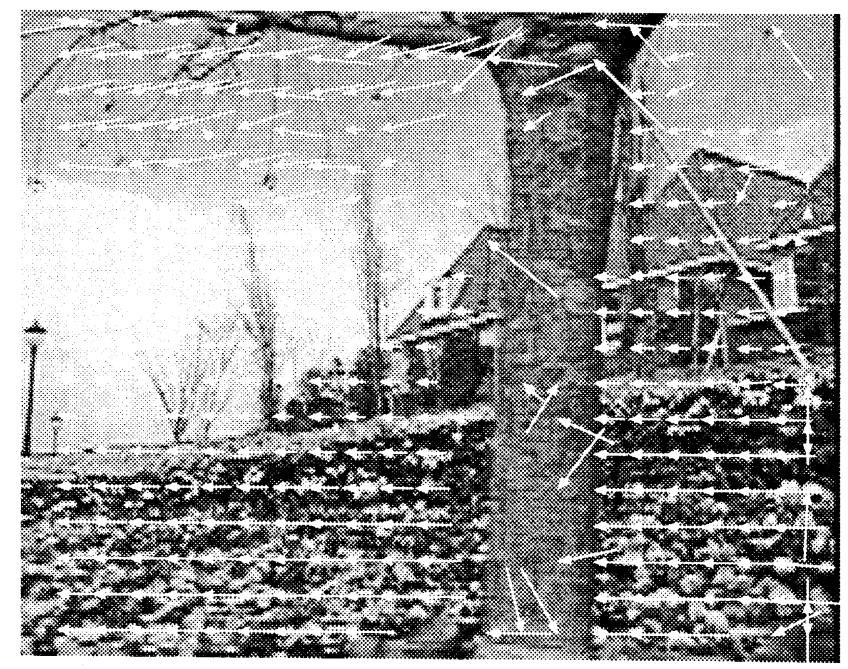

Fig. 1 An example of global motion.

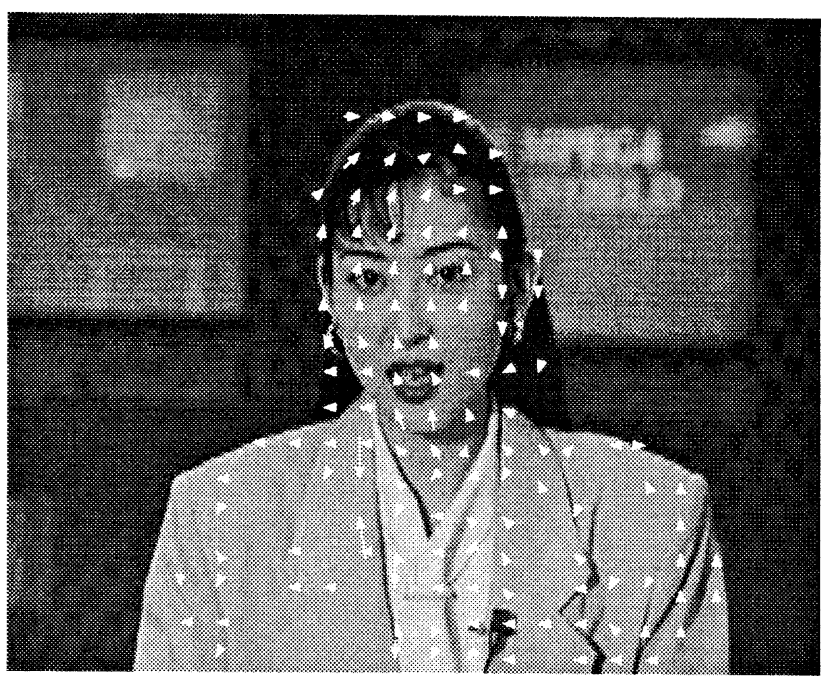

Fig. 2 An example of local motion.

\section{Detection in Frames}

In order to extract the sequences of each type of camera work from a video, the algorithm analyzes only Bframes and P-frames, in MPEG2 encoded data, I-frames do not contain any motion vector.

\section{1 Camera Operations}

Regular camera operations include panning, tilting, zooming, tracking, booming and dollying together with the many combinations of these operations that are possible. The six basic camera operations used in this paper are shown in Fig.3. To simplify the following discussion, panning and tracking operations, tilting and booming operations, and zooming and dollying operations are considered to be the same camera operations. Furthermore, tilting up and tilting down are hereafter referred to as panning up and panning down.

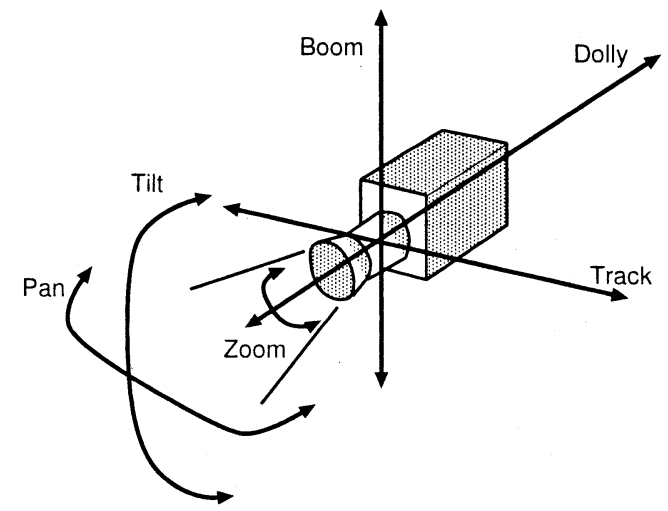

Fig. 3 Basic camera operations.

\section{2 Detection Scheme}

An algorithm that captured the motion vectors in one frame by dividing the picture into four parts has been proposed ${ }^{3)}$. However, its average detection rate was only $70 \%$ and was lower than $70 \%$ when the video was lively, because it calculated the overall motion vectors and so contained local motion. Since camera work generates the global motion, if the global motion can be extracted, it will be easy to detect the camera work. However, it is difficult for computers to partition the global motion and local motion in one frame on the basis of analyzing various pattens of motion vectors.

To solve this problem and improve the detection rate, this paper divided the picture into 9,16 or 25 to compare the results using the same test data (See Fig.4). Finally, this paper chooses the nine division method and calculates the motion vectors only in the four corners because it is obvious that the objects generating local motion are often at the center of the picture. This 
scheme can suppress the extraction of local motion as much as possible while providing enough motion vectors to analyze the camera work. Three essential factors are used to decide the camera work. The first is the direction of each motion vector, the second is the length of each motion vector, and the third is number of the motion vectors having the same direction.

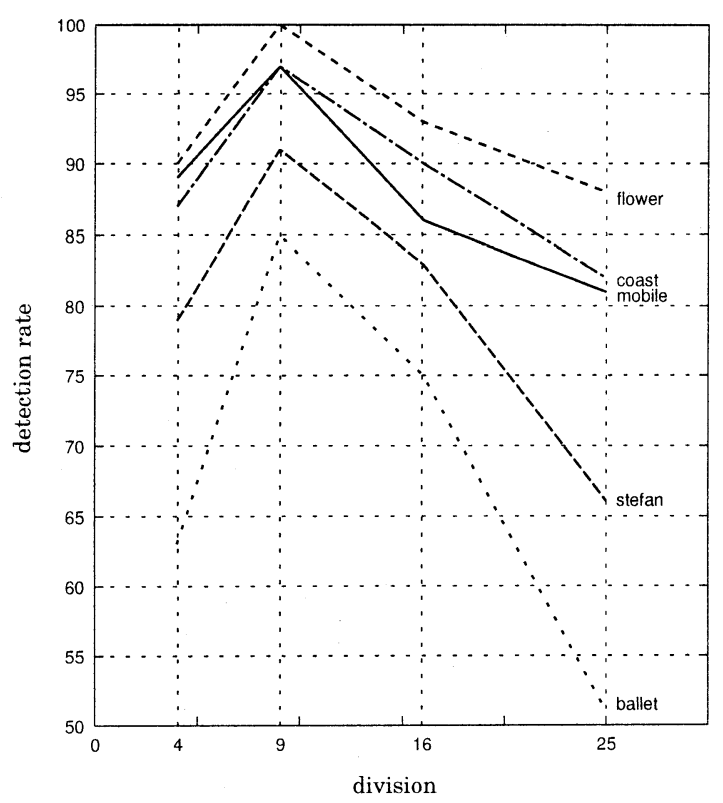

Fig. 4 The detection rate of data in different division.

\subsection{Panning Detection}

As mentioned above, the picture is divided into nine parts. For detecting the directions of panning such as left, right, up and down, the motion vectors in the four parts of corner are processed into four angle parts according to their angles. The four angle parts are shown as follows:

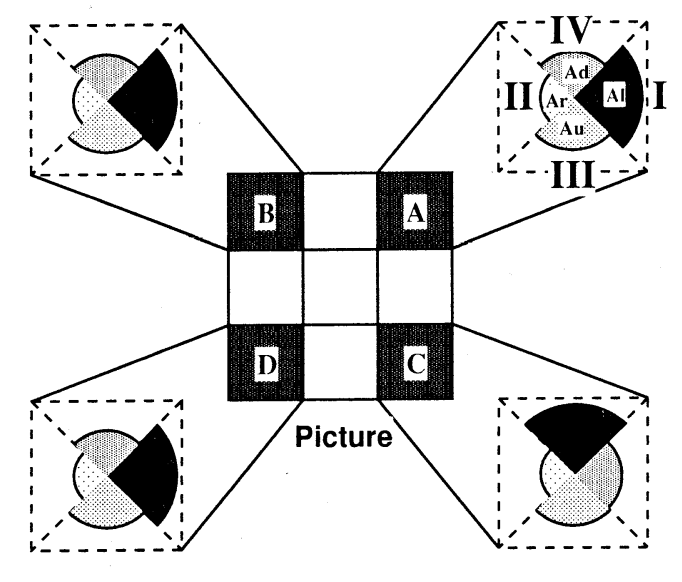

Fig. 5 An example of pan left decision.

$$
\begin{array}{cc}
\text { the part of } I: & 45^{\circ}>\alpha \text { or } \alpha \geqq 315^{\circ} \\
\text { the part of } I I: & 135^{\circ} \leqq \alpha<225^{\circ}
\end{array}
$$

$\begin{aligned} \text { the part of } I I I: & 225^{\circ} \leqq \alpha<315^{\circ} \\ \text { the part of } I V: & 45^{\circ} \leqq \alpha<135^{\circ}\end{aligned}$

where, $\alpha$ is the motion vector angle. For the picture, the camera work mode can be recognized as pan left, right, up, or down while the four maximum numbers of motion vectors in the four corners belong to the part of I, II, III, or IV, respectively.

In this nine division method, the four maximum numbers of the motion vectors, which exceed a certain length, in corners $A, B, C$ and $D$ are calculated to decide the panning mode of the picture (See Fig.5). In corner $A$, the magnitudes of the fan-shaped area of $A l$, $A r, A u$ and $A d$ are used to indicate the number of motion vectors that exceed a certain length in the four angle parts of $I, I I, I I I$, and $I V$. The maximum number is then found from $A l, A r, A u$ and $A d$. The corresponding nomenclature is used for corners $B, C$ and $D$. So there is a maximum number in each of corner $B, C$ or $D$, too. $P l, P r, P u$ and $P d$ are expressed as follows: $P l=A l+B l+C l+D l, P r=A r+B r+C r+D r$, $P u=A u+B u+C u+D u, P d=A d+B d+C d+D d$. The panning mode is recognized when at least on of the following conditions are fulfilled:

-More than three maximum numbers of the four corners lie in the same angle part of $I, I I, I I I$, or $I V$.

-The two maximum numbers of the four corners lie in the same angle part, and the other two maximum numbers of the four corners are in a different angle part.

-The sum of the same angle part in all four corners is maximum $P_{\max }$ by comparison with the other three angle parts, where $P_{\max }=\max \{P l, P r, P u, P d\}$.

For example, figure 5 describes the panning left decision that fulfills the first condition above. $A l, B l$, and $D l$ are the maximum numbers in corners $A, B$, and $D$, respectively, and they are in the same angle part of $I$. Panning left mode is recognized in this angle part. In the same way, panning right, up, or down mode can be recognized in the angle parts of $I I, I I I$, or $I V$.

\section{4 Zoom Detection}

The motion vectors are angled toward the outside of the picture in the case of zoom in and toward the center of the picture in the case of zoom out.

As for zoom detection, the picture is also divided into nine parts and only the four corners are processed, but the motion vectors are divided into different four angle parts. The four angle parts are shown as follows:

$$
\begin{aligned}
\text { the part of } i: & 0^{\circ} \leqq \beta<90^{\circ} \\
\text { the part of } i i: & 90^{\circ} \leqq \beta<180^{\circ}
\end{aligned}
$$


the part of iii : $\quad 180^{\circ} \leqq \beta<270^{\circ}$

the part of iv : $270^{\circ} \leqq \beta<360^{\circ}$

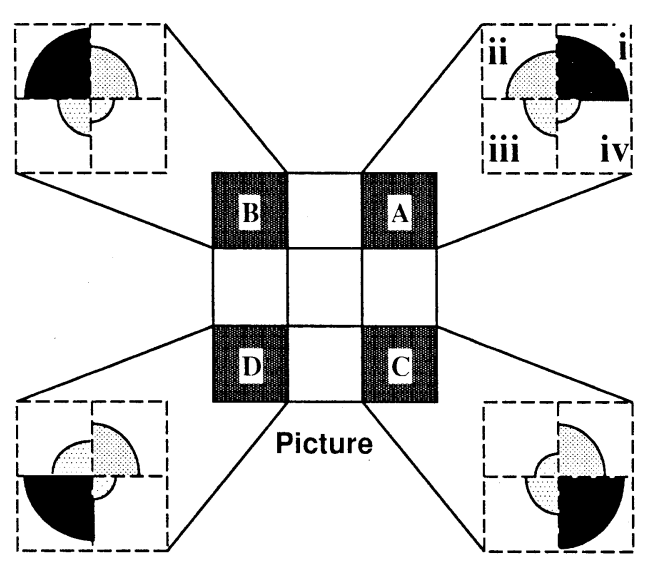

Fig. 6 Zoom in detection.

Similar to panning mode decision, zoom in (zoom out) mode is recognized if the maximum number of motion vectors belong to part $i$ (part iii) in corner $A$, part $i i$ (part $i v$ ) in corner $B$, part $i i i$ (part $i$ ) in corner $C$, and part $i v$ (part $i i$ ) in corner $D$ (See Fig.6). In this paper, zoom in or zoom out mode can be recognized when more than three maximum numbers are in the regular angle parts.

Table 1 The parameters of test video sequences

\begin{tabular}{c|c}
\hline \hline mpeg2 sequence factor & parameters \\
\hline frame rate & 30 frames $/ \mathrm{sec}$ \\
\hline frames in GOP & 15 frames \\
\hline I/P frame distant & 3 frames \\
\hline picture format & frame picture coding \\
\hline horizontal size & 704 pixeles $^{*}$ \\
\hline vertical size & 480 lines $^{* *}$ \\
\hline bit rate & $5.4 \mathrm{M} \mathrm{bits/sec}$ \\
\hline$* 352$ pixeles for standard video data \\
** 288 lines for standard video data
\end{tabular}

\subsection{Results in Frames}

This algorithm was implemented by and tested with several kinds of MPEG2 compressed video sequences. We first report the results of camera work detection in each frame, and then the pattern of these results will be used as data for deciding the sequences of camera work in the video sequence. Standard MPEG2 encoded video data were used in these experiments as well as some test video sequences encoded by a MPEG2 hardware encoder system from TV programs containing panning and zooming. The parameters of the test video sequences are shown in Table 1.
Table 2 shows the results of test video data in each frame (except I-frames) in a comparison with manual decision.

The experiment used 2514 frames (except I-frames) of the test video data. 2154 frames of camera work were detected correctly. From Table 2, the detection rate of camera work in each frame is above $80 \%$ (except I-frames); the lowest and the average detection rate is about $85.7 \%$ ( $2154 / 2514)$.

Table 2 Experimental results in each frame

\begin{tabular}{|c|c|c|c|c|c|c|c|c|c|c|c|c|c|}
\hline \multirow{2}{*}{$\begin{array}{c}\text { video } \\
\text { sequences }\end{array}$} & \multirow{2}{*}{$\begin{array}{c}\text { frame } \\
\text { number }\end{array}$} & \multicolumn{2}{|c|}{ pan left } & \multicolumn{2}{|c|}{ pan right } & \multicolumn{2}{|c|}{ tilt up } & \multicolumn{2}{|c|}{\begin{tabular}{|l} 
tilt down \\
\end{tabular}} & \multicolumn{2}{|c|}{ zoom in } & \multicolumn{2}{|c|}{ zoom out } \\
\hline & & $\mathrm{V}$ & D & $\mathrm{V}$ & D & $\mathrm{V}$ & D & $\mathrm{V}$ & D & V & D & $\mathrm{V}$ & D \\
\hline mobile & 150 & \begin{tabular}{|l|}
150 \\
\end{tabular} & 136 & & & & & & & & & & \\
\hline ballet & 150 & 59 & 50 & 91 & 68 & & & & & & & & \\
\hline flower & 150 & & & 150 & 139 & & & & & & & & \\
\hline stefan & 300 & 151 & 127 & 130 & 111 & 11 & 9 & & & 8 & 7 & & \\
\hline coast & 300 & 64 & 57 & 225 & 205 & 11 & 8 & & & & & & \\
\hline table-t & 150 & & & 14 & 12 & & & & & & & 43 & 40 \\
\hline skate 1 & 170 & 39 & 34 & 119 & 95 & & & & & 12 & 11 & & \\
\hline skate2 & 192 & 73 & 61 & 119 & 98 & & & & & & & & \\
\hline skate3 & 256 & 77 & 57 & & & 49 & 36 & 30 & 25 & 100 & 82 & & \\
\hline lab. & 240 & 29 & 23 & 36 & 34 & 47 & 36 & 31 & 29 & 53 & 47 & 44 & 38 \\
\hline tree & 281 & 106 & 84 & & & & & 175 & 146 & & & & \\
\hline cave & 355 & & & 139 & \begin{tabular}{|l|}
102 \\
\end{tabular} & & & 13 & 11 & 184 & \begin{tabular}{|l|}
136 \\
\end{tabular} & & \\
\hline
\end{tabular}

\section{Camera Work Sequence Extraction}

As mentioned before, the camera work of each frame was detected from MPEG2 compressed video sequences and the average detection rate is above $85 \%$. A method of extracting the camera work sequences in a video sequence that calculates the beginning and the end frame number of each camera work is proposed below.

\subsection{Basic Scheme}

Because there will always be some uncertainty in deciding which camera work is dominant in a sequence, only two patterns are extracted: consecutive and interval patterns. A key parameter is the length of continuous camera work used to define a visually stable and coherent piece of camera work. Based on extensive research we set this length at 5 frames.

\section{2 Consecutive Pattern}

A consecutive pattern is a segment running from the first instance of five consecutive frames with the same type of camera work to the last frame of the same type of camera work that immediately precedes the next set of five consecutive frames of a different type of camera work.

Fig.7 shows an example of a consecutive pattern and processing results.

In Fig.7, the segment from frames 29 to 48 , is recognized as pan left based on the definition of the consecutive pattern, and the beginning and the end frame 
number is saved.

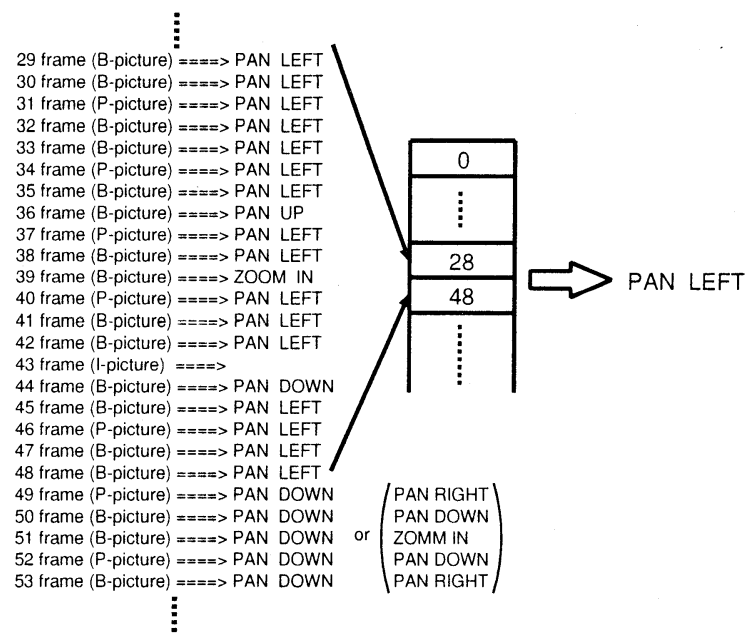

Fig. 7 An example of consecutive pattern processing.

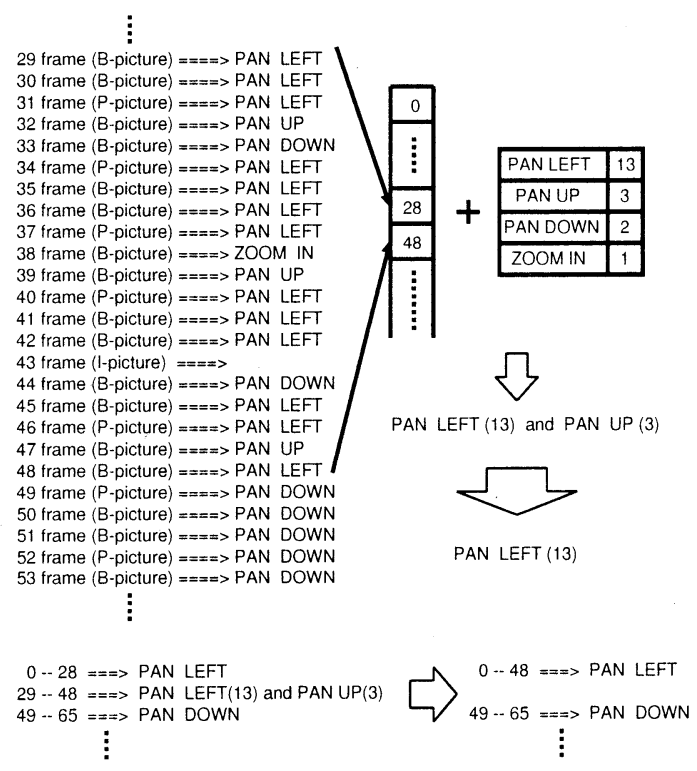

Fig. 8 An example of interval pattern processing.

\section{3 Interval Pattern}

An interval pattern is a segment running from the end of one consecutive pattern to the beginning of the next consecutive pattern.

The numbers of each type of camera work in the interval pattern are calculated and are arranged in magnitude. The two most frequent types of camera work are determined and hereafter we refer to the frequency counts as $A$ and $B$, in descending order of frequency. The interval pattern is only ascribed to $A$ if the expression of $n \leqq A / B$ is fulfilled, where $n$ is the ratio of $A$ to $B$, otherwise it will be described as the combined $A$ and $B$. For determining the value of $n, 1.5,2$, or 3 is attempted with the several kinds of video materials. The judgement is the most correct at $n=2$ in above three values.

When the two types of camera work are contradictory, for example, pan left and pan right, the extraction is deemed to have failed and the pattern is dropped.

The Fig.8 shows an example of the processing of an interval pattern. The segment from frames 29 to 48 is defined as an interval pattern, and the frequency counts of each type of camera work are calculated. The two most frequent types are "Pan left(13)" and "Pan up(3)". Finally, this sequence is recognized as "Pan left" because the number of "Pan left(13)" is more than twice that of "Pan up(3)". This makes the description of camera work more accurate.

\section{4 Processing Flow}

The processing flow of camera work sequence extraction is shown in Fig.9.

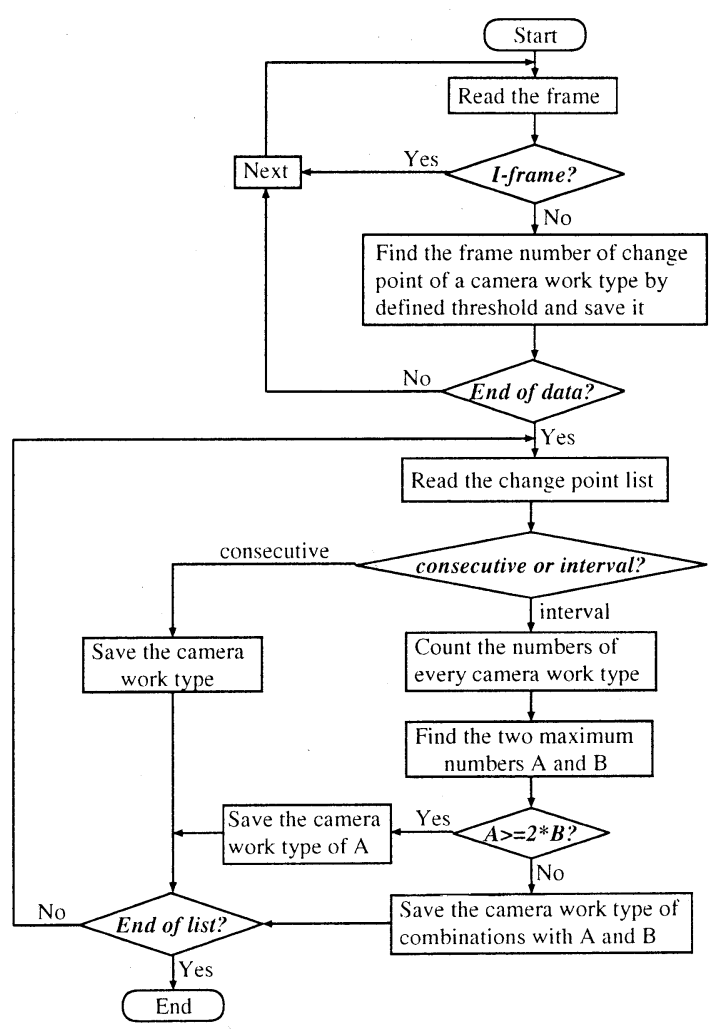

Fig. 9 The processing flow of the proposed algorithm.

\section{Results}

\section{1 Extraction Rate}

Camera work sequence extraction was tested using the detection algorithm mentioned in section 2 and section 3. Twelve MPEG2 video data sets were processed by the proposed algorithm. The extraction rate for the 12 videos was closed to $100 \%$ (See Table 3 ). 
Table 3 The results of camera work sequences extraction

\begin{tabular}{|c|c|c|c|}
\hline $\begin{array}{l}\text { Video } \\
\text { data }\end{array}$ & Camera work by Vision & $\begin{array}{l}\text { Extrac- } \\
\text { tion rate }\end{array}$ & Note \\
\hline mobile & Left & $100 \%$ & -- \\
\hline ballet & Right -> Left & $100 \%$ & -- \\
\hline flower & Right & $100 \%$ & $\because$ \\
\hline stefan & $\begin{array}{l}\text { Right }->\text { Up } \rightarrow \text { Left }-> \\
\text { Right } \rightarrow \text { Left }->\text { In }\end{array}$ & $100 \%$ & -- \\
\hline coast & Left $>$ Up $>>$ Right & $100 \%$ & -- \\
\hline table-t & Static $->$ Out $>>$ Static $->$ Right & $100 \%$ & -- \\
\hline skate 1 & Right $->$ In $->$ Left & $98 \%$ & $\begin{array}{l}\text { There are some blurring frames } \\
\text { in the "Pan right" and "Zoom in" }\end{array}$ \\
\hline skate2 & Right $->$ Left & $100 \%$ & -- \\
\hline skate3 & In and Down $>$ Left $\rightarrow$ Up & $97 \%$ & $\begin{array}{l}\text { "Zoom in" and "Pan down" are } \\
\text { respectively detected by algriothm }\end{array}$ \\
\hline lab. & $\begin{array}{l}\text { Left } \rightarrow \text { Right } \rightarrow>\text { Up } \rightarrow> \\
\text { Down } \rightarrow \text { In } \rightarrow>\text { Out }\end{array}$ & $99 \%$ & $\begin{array}{l}\text { There are some blurring frames } \\
\text { in the "Pan left" and "Pan right" }\end{array}$ \\
\hline tree & Down $\rightarrow$ Down and Left $>$ Left & $98 \%$ & $\begin{array}{l}\text { "Pan down" and "Pan left" are } \\
\text { respectively detected by algriothm }\end{array}$ \\
\hline cave & In $->$ Static $->$ Right and Down & $100 \%$ & - \\
\hline
\end{tabular}

\section{2 Application System}

A system for video editing was developed around the proposed algorithm. A screen shot is shown in Fig.10. For editing purposes, only the first frame of every camera work sequence is decoded and displayed in this system. This allows the user to more fully understand the contents of an MPEG2 encoded video more quickly.

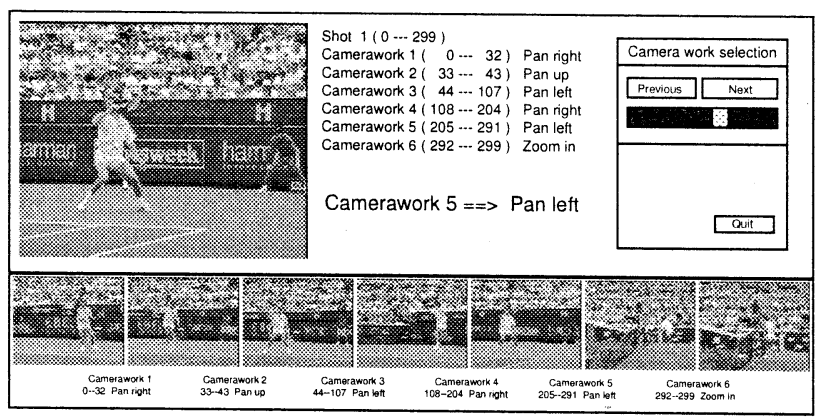

Fig. 10 The system for video editing and understanding.

\section{Conclusion}

By using motion vectors, an efficient and a practical method has been developed to process MPEG2 compressed video sequences; It extracts camera work sequences for use in video editing systems. The algorithm proposed herein can successfully handle all $\mathrm{P}$ frames and B-frames, it can also find the beginning and the end frame number of each pattern in the video. In an experiment, closed to $100 \%$ of various camera work sequences were successfully extracted even from some lively video data. However, the length of key parameter and the ratio $n$ in section 3.3 may be decided further by much more video data again. This method reduces the volume of processing by about $50 \%$ compared to the four division method because the proposed nine division method treats only $4 / 9$ of the picture.

Future work includes studying more robust camera work detection algorithms with clear differentiation between noise and motion. It will be important to devise an algorithm to partition the global motion and local motion and a scheme for distinguishing panning from tracking, tilting from booming, and zooming from dollying. Moreover, the detection rate for camera work while the moving objects occupying a large area of the picture or the scene changes are complex such as fades, dissolves, and wipes must be improved.

It will become possible to interpolate the loss frames from $\mathrm{P}$-frames in MPEG2 encoded data transmision if $\mathrm{P}$-frames were related to $\mathrm{B}$-frames. This relation can be confirmed by camera work sequence extraction. Furthermore, it would become possible to extract the blurred frames that can not be handled by existing video editing tools.

\section{[References]}

1) Y.Tonomura, et al.: "Structured video computing", IEEE Multimedia, pp. 34-43 (Fall 1994)

2) Y.Yang, S.Nakano, M.Dosho: "A Study on Camera Work Detection from MPEG2 Encoded Data", JCIS CVPRIP'98, pp. 246-249 (1998)

3) S.Nakano, Y.Yang and K.Kawashima: "A Study on a Video Editing System that Uses the Broadband Computer Network", IEEE ISCE'97, pp. 267-271 (1997)

4) A.Akutsu, et al.:"Video Indexing Using Motion Vectors", Proc. SPIE Visual Comm. and Image Processing 92, SPIE, Bellingham, Wash., Vol. 1818, pp.1522-1530 (1992)

5) Hotter M.: "Differential Estimation of the Global Motion Parameters Zoom and Pan", Signal Processing, 16, 3, pp. 249-265 (Mar. 1989)

6) A.Akutsu, et al.:"Video Tomography: An Efficient Method for Camerawork Extraction and Motion Analysis", Proc. of ACM Multimedia 94, pp.349-356 (1994)

7) Kamikurs K. and Watanabe H.: "A Study on Pan and Zoom Compensation for Picture Coding", 1990 Spring Natl. Conv. Rec. IEICE, D-299

8) Tse Y.T. and Baker R.L.: "Global Zoom/Pan Estimation and Compensation for Video Vompression", IEEE ICASSP'91, pp. 2725-2728 (1991)

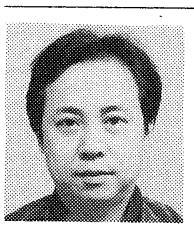

Yang Yang was born in Dalian, China. He received his B.S. degree from Guizhou University, China, in 1984. He was an assistant of the Computer Department at Dalian University in China. Since 1991, he has been engaged in the Compute Center of Liaoning Foreign Trade Bureau in China. $\mathrm{He}$ is currently a postgraduate student of Toyama Prefectural University in Japan.

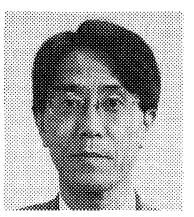

Nakano Shizuo was born in Toyama, Japan. He received his M.E. degree from Kanazawa University in 1973, and his Ph.D degree from the University of Tokyo in 1992. He joined the NTT Electrical Communications Laboratories (ECL), Nippon Telegraph and Telephone Corporation, in 1973, and commenced researching and developing communication terminals and multimedia systems. Since 1995 , he has been a Professor at Toyama Prefectural University, Toyama, Japan. His research interests include computer networking, multimedia communication systems, and human interface design. 